\title{
Eriocitrin attenuates ischemia reperfusion-induced oxidative stress and inflammation in rats with acute kidney injury by regulating the dual-specificity phosphatase 14 (DUSP14)-mediated

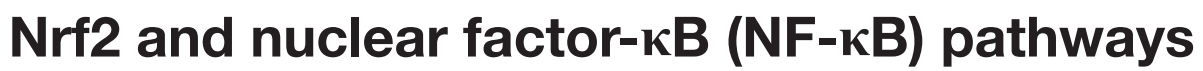

\author{
Jun $\mathrm{Xu}^{1,2}$, Liang $\mathrm{Ma}^{1}$, Ping $\mathrm{Fu}^{1}$ \\ ${ }^{1}$ Division of Nephrology and National Clinical Research Center for Geriatrics, Kidney Research Institute, West China Hospital of Sichuan \\ University, Chengdu, China; ${ }^{2}$ Division of Nephrology, The Affiliated Baiyun Hospital of Guizhou Medical University, Guiyang, China \\ Contributions: (I) Conception and design: J Xu, L Ma; (II) Administrative support: P Fu; (III) Provision of study materials or patients: L Ma, P Fu; (IV) \\ Collection and assembly of data: J Xu, L Ma; (V) Data analysis and interpretation: Ping Fu; (VI) Manuscript writing: All authors; (VII) Final approval \\ of manuscript: All authors. \\ Correspondence to: Liang Ma; Ping Fu. Division of Nephrology, Kidney Research Institute, West China Hospital of Sichuan University, No.37 Guoxue \\ Alley, Wuhou district, Chengdu 610041, China. Email: ut779224@21cn.com; pingfudoc@126.com.
}

Background: Ischemia reperfusion (IR)-induced acute kidney injury (AKI) is accompanied by increased inflammatory response and oxidative stress. Eriocitrin is a flavonoid that is mainly derived from lemon or citrate juice. It exhibits various pharmacological effects and is known to have antioxidant and anti-steatotic benefits. However, research on the effect of eriocitrin against IR-induced oxidative stress and inflammation in AKI is limited.

Methods: In this study, an OGD/R of HK-2 cell in vitro and rat model of AKI in vivo were constructed. Then the cell or rats were treated with eriocitrin at different doses $(60,30,10 \mathrm{mg} / \mathrm{kg})$. The levels of apoptotic were detected by flow cytometry. Inflammatory and oxidative stress factors in supernatant in vitro and tissue in vivo. Meanwhile, Western blot was used to detect the change of dual-specificity phosphatase 14 (DUSP14), Nrf2 and nuclear factor- $\kappa \mathrm{B}(\mathrm{NF}-\kappa \mathrm{B})$.

Results: Eriocitrin attenuated apoptosis of the human renal tubular epithelial cell line HK-2 mediated by oxygen glucose deprivation/reperfusion via the repression of inflammation and oxidative stress in a dosedependent manner. Eriocitrin also enhanced the levels of dual-specificity phosphatase 14 (DUSP14) and Nrf2, and decreased NF- $\kappa$ B phosphorylation. Furthermore, the in vivo experiments indicated that eriocitrin dose-dependently alleviated IR-induced AKI and apoptosis in rats. By elevating DUSP14, eriocitrin promoted the expression of Nrf2 and inactivated NF- $\mathrm{BB}$, thereby downregulating inflammation and oxidative stress. Moreover, inhibiting DUSP14 expression with protein tyrosine phosphatase (PTP) inhibitor IV reversed the kidney-protective effects of Eriocitrin.

Conclusions: Eriocitrin protected IR-induced AKI by attenuating oxidative stress and inflammation via elevating DUSP14, thereby providing a theoretical basis for the treatment of IR-induced AKI.

Keywords: Acute kidney injury (AKI); eriocitrin; oxidative stress; inflammation; dual-specificity phosphatase 14 (DUSP14)

Submitted Dec 24, 2020. Accepted for publication Feb 08, 2021.

doi: $10.21037 / \mathrm{atm}-21-337$

View this article at: http://dx.doi.org/10.21037/atm-21-337 


\section{Introduction}

Acute kidney injury (AKI) is one of the most common and serious complications following ischemia reperfusion (IR). AKI is characterized by acute renal failure (ARF), which manifests as insufficient blood filtration and disorders of water production, ion regulation, and urine (1). As research on IR has deepened, a growing number of studies have testified that the onset and progression of IR-induced AKI are accompanied by expressive alterations and abnormal functioning of various genes, ribonucleic acids (RNAs), and proteins (2). It is also notable that inflammatory response and oxidative stress play key roles in AKI (2). Inflammatory cytokines and oxidative stress factors could aggravate the secondary injury in AKI, leading to the destruction of renal tubules and glomeruli (2).

In recent years, numerous bioactive compounds of natural origin have attracted attention as hotspots in drug research and development, and new drugs have consequently been discovered and developed for the treatment of various diseases. Eriocitrin is a flavonoid compound mainly derived from lemon or citrate juice. It is known to exert protective effects on human health (3), and to possess anti-diabetic, anticarcinogenic, antioxidative, and anti-steatotic pharmacological benefits (4). Some scholars have reported that eriocitrin also attenuates oxidative damage in rat liver tissues (5). As a consequence, the biological activity of eriocitrin as well as its mechanism of action and pharmacokinetics in IR have become hot research topics in recent years. However, no systematic claims or evidence of the curative effects of eriocitrin against IR-induced oxidative stress and inflammation in AKI has been presented in previous reports.

Dual-specificity phosphatase 14 (DUSP14), also known as MKP6, contains a C-terminal catalytic domain, which is consistent with other DUSP family members, although it lacks the $\mathrm{N}$-terminal $\mathrm{CH} 2$ domain and is an atypical DUSP (6). DUSP14 dephosphorylates mitogen-activated protein kinases (MAPKs), including signal molecules such as extracellular regulated protein kinases (ERK)1/2, p38, and c-Jun $\mathrm{N}$-terminal kinase (JNK), thereby regulating diverse cellular responses such as differentiation, stress response, growth, and immune defense $(7,8)$. IR directly activates JNK and $\mathrm{p} 38$, thus eventually activating nuclear factor- $\mathrm{KB}(\mathrm{NF}-\kappa \mathrm{B})$ and affecting the DUSP activity. In contrast, overexpression of DUSP14 inhibits NF- $\kappa \mathrm{B}$ activation triggered by tumor necrosis factor- $\alpha(\mathrm{TNF}-\alpha)$ and interleukin $1 \beta$ (IL-1 $\beta)$, thereby restraining the inflammatory response $(9,10)$. Furthermore, antioxidant activity is also inhibited by DUSP14. For instance, Yang et al. confirmed that DUSP14-knockout (KD) mice exhibited strengthened immune responses and were more susceptible to experimental autoimmune encephalomyelitis (7). Meanwhile, Song et al. reported that DUSP14 overexpression notably reduces the production of reactive oxygen species (ROS), and downregulates TNF- $\alpha$ and IL- $1 \beta$ via upregulation of the Nrf-2 signaling pathway, thereby protecting mice from IR-induced brain damage $(11,12)$.

Our previous study revealed that eriocitrin (Figure S1) relieved oxygen glucose deprivation/reperfusion (OGD/ $\mathrm{R}$ )-induced renal tubular epithelial cell injury with antiapoptotic, anti-inflammatory, and antioxidative stress effects. Meanwhile, eriocitrin was found to promote Nrf2 and DUSP14 expression while inactivating the NF$\kappa \mathrm{B}$ pathway. Hence, in the present study, we performed further experiments to investigate the therapeutic effects of eriocitrin in an experimental animal model of IR-induced AKI. We present the following article in accordance with the ARRIVE reporting checklist (available at http://dx.doi. org/10.21037/atm-21-337).

\section{Methods}

\section{Experimental kidney IR model and grouping}

Adult male Sprague-Dawley (SD) rats (6-8 weeks, 220-250 g, 60 rats) were supplied by the Experimental Animal Center of West China Hospital of Sichuan University. The rats were kept under 12-hour light/12-hour dark standard conditions with free access to food and water. The method used to establish the model was as follows. Briefly, the back hair of rats in the sham group was shaved under anesthesia, the skin was cleaned and disinfected with povidone-iodine, and then surgery was performed. The incision was stitched with $3 / 0$ silk sutures, and no IR model or drug treatment was given after surgery for sham group. In the IR group, the bilateral kidney arterial and venous of the rats were clamped with a non-invasive microvascular clamp after exposure. After 1 hour, the clamp was opened to recirculate the blood for 24 hours for reperfusion. The remaining steps were consistent with those of the sham group. After that, the kidney tissues were removed from the rats for subsequent experiments. All steps were carried out under intraperitoneal anesthesia of xylazine hydrochloride (Rompun, Bayer, Istanbul) at a dose of $10 \mathrm{mg} / \mathrm{kg}$ body weight and ketamine (Ketalar, Pfizer, Istanbul) at a dose of $15-60 \mathrm{mg} / \mathrm{kg}$ body 
weight. All animal experiments were approved by the Ethics Committee of The Affiliated Baiyun Hospital of Guizhou Medical University. All animal operations were performed according to the National Institutes of Health Guide for the Care and Use of Laboratory Animals.

After adaptive rearing, 60 rats were weighed and randomly divided into 6 groups according to their weight, with 10 in each group. The 6 groups were: sham group, IR group, high-dose group (eriocitrin $60 \mathrm{mg} / \mathrm{kg}$ ), mediumdose group (eriocitrin $30 \mathrm{mg} / \mathrm{kg}$ ), low-dose group (eriocitrin $10 \mathrm{mg} / \mathrm{kg})$, and eriocitrin $(60 \mathrm{mg} / \mathrm{kg})+$ protein tyrosine phosphatase (PTP) inhibitor IV group. Eriocitrin was intraperitoneally injected once a day, from the day of modeling until the end of the experiment. The DUSP14specific inhibitor PTP inhibitor IV (Calbiochem, San Diego, CA, United States) inhibitor was administered via tail vein injection once a day, $5 \mathrm{mg} / \mathrm{kg}$ each time, from the day of modeling until the end of the experiment.

\section{Cell culture and grouping}

The human renal tubular epithelial cell line HK-2 was purchased from the cell bank of the Chinese Academy of Sciences. HK-2 cells were stored in Dulbecco's modified eagle medium (DEME) medium (Invitrogen, Carlsbad, CA, United States) supplemented with L-glutamine (Gibco, New York, CA, USA), $100 \mathrm{U} / \mathrm{mL}$ penicillin and $100 \mathrm{mg} / \mathrm{mL}$ streptomycin (Sinopharm Chemical Reagent, Beijing, China). Then, the cells were incubated in an incubator with $5 \% \mathrm{CO}_{2}$ at $37^{\circ} \mathrm{C}$.

Cell grouping and treatment: Control group: no treatment was given. OGD/R group: treated according to the OGD/R model establishment method. Eriocitrin groups: before OGD/R treatment, HK-2 cells were treated with different doses of eriocitrin $(1,2$, or $4 \mu \mathrm{M} / \mathrm{mL})$ for 30 minutes, and eriocitrin was continuously administered at the same dose as the initial until the end of the experiment. In addition, the DUSP14-specific inhibitor, PTP inhibitor IV (50 $\mu$ M, San Diego, CA, United States), treatment started at 30 minutes before OGD/R treatment and ended at the end of the experiment.

\section{Establishment of an in vitro OGD/R cell model}

An in vitro OGD/R cell model was established as follows. After 6 days of culture, the human renal tubular epithelial cell line HK-2 was washed 3 times with glucose-free Dulbecco's Modified Eagle Medium (DMEM, Gibco,
Carlsbad, CA, United States), which had previously been balanced in an incubator, supplemented with $1 \% \mathrm{O}_{2}, 5 \%$ $\mathrm{CO}_{2}$, and $94 \% \mathrm{~N}_{2}$ at $37^{\circ} \mathrm{C}$. Then, the original medium was replaced, and the cells were transferred to an incubator containing $1 \% \mathrm{O}_{2}, 5 \% \mathrm{CO}_{2}$, and $94 \% \mathrm{~N}_{2}$, and maintained at $37^{\circ} \mathrm{C}$ for 1.5 hours. Subsequently, the medium was changed back to the original medium, and the culture was returned to the normal incubator with a recovery time of 6 hours. Similarly, the control cells were cultured in neural basal medium in an incubator with $5 \% \mathrm{CO}_{2}$ for the same amount of time, with the $\mathrm{pH}$ of the medium maintained at 7.2. Finally, after 24 hours, the cell viability was measured.

\section{Quantitative real-time polymerase chain reaction}

Primers were designed with Primer 3.0 software (primer corporation, Canada) according to the sequence number of each gene in GenBank. Total RNA was extracted from kidney tissues and HK-2 cells using TRIzol reagent (Invitrogen). Moloney Murine Leukemia Virus (M-MLV) reverse transcriptase (Invitrogen) was applied for reverse transcription of total RNA into cDNA. Quantitative realtime polymerase chain reaction (qRT-PCR) was performed on an ABI 7500 real-time PCR system (Applied Biosystems, San Francisco, CA, United States) using SYBR Premix Ex Taq II (TaKaRa, Dalian, China) according to the manufacturer's instructions as follows: pre-denaturation at $95^{\circ} \mathrm{C}$ for 10 minutes, then $95^{\circ} \mathrm{C}$ for 15 seconds and $60{ }^{\circ} \mathrm{C}$ for 15 seconds, for a total of 45 cycles, with the fluorescence signal temperature as $60^{\circ} \mathrm{C}$. Glyceraldehyde 3-phosphate dehydrogenase (GAPDH) was used as the standardized internal reference for IL-1 $\beta$, while U6 served as that for miR-152-3p. Gene expression was calculated using the $2^{-\Delta \Delta \mathrm{Ct}}$ method. Each experiment was repeated 3 times, and the measurements were performed 3 times. The specific primer sequences used were in Table 1.

\section{Flow cytometry}

An Annexin V-FITC Apoptosis Detection Kit (Beyotime, Shanghai, China) was used to detect apoptosis of HK-2 cells. Firstly, HK-2 cells in the logarithmic growth phase were trypsinized with $0.25 \%$ trypsin (Beyotime, Shanghai, China) and then seeded into 6-well culture plates with $1 \times 10^{6}$ cells/well. After completion of the treatments, the cells were collected and resuspended with cold phosphatebuffered saline (PBS). Then, approximately $1.5 \times 10^{5}$ cells were taken and centrifuged $\left(1,500 \mathrm{r} / \mathrm{min}, 4^{\circ} \mathrm{C}\right)$, after which 
Table 1 The sequences of primers

\begin{tabular}{lrr}
\hline Gene & Forward & Reverse \\
\hline TNF- $\alpha$ & 5'-AACACGCGCTGACTCCTAGT-3' & 5'-CAGTGCAGGGTCCGAGGT-3' \\
IL-1 $\beta$ & 5'-TTCAACCTGCATCCTACCCC-3' & 5'-GAGAGACAGATCCCGGAGAC-3' \\
Interleukin-6 (IL-6) & 5'-CCCCGTAGATTGCAAACTCC-3' & 5'-TGTCCTTGCCAGTGTCTTCT-3' \\
DUSP14 & 5'-GCTTCGGCAGCACATATACTAAAAT-3' & 5'-CGCTTCACGAATTTGCGTGTCAT-3' \\
GAPDH & 5'-TATGATGATATCAAGAGGGTAGT-3' & 5'-TGTATCCAAACTCATTGTCATAC-3' \\
\hline
\end{tabular}

the supernatant was discarded, and $400 \mu \mathrm{L}$ of $1 \times$ Annexin $\mathrm{V}$ Binding Buffer was added to gently resuspend the cells. Finally, the cells were incubated at room temperature for 20 minutes in darkness, and $5 \mu \mathrm{L}$ propidium iodide (PI) was added and mixed with the cells gently. After that, flow cytometry was performed within 1 hour (excitation wavelength $\mathrm{Ex}=494 \mathrm{~nm}$; emission wavelength $\mathrm{Em}=535 \mathrm{~nm}$ ).

\section{Cell Counting Kit-8 assay}

Cell viability was determined according to the instructions of the Cell Counting Kit-8 (CCK-8) kit (Dojindo, Kumamoto, Japan). Firstly, HK-2 cells were seeded in 96well plates, with the cell density adjusted to $5 \times 10^{3} / \mathrm{mL}$. Then, after adherence, the cells were treated according to the previous describe about the dividing groups. Following that, $10 \mu \mathrm{L}$ enhanced CCK-8 solution was added and incubated at $37{ }^{\circ} \mathrm{C}$ for 1 hour. Finally, the absorbance was measured at $450 \mathrm{~nm}$. Each experiment was repeated 3 times, and the measurements were performed 3 times.

\section{Western blot}

After their different treatments, kidney tissues or cells were collected. Total protein was extracted through the addition of RIPA lysate (Beyotime Biotechnology, Shanghai, China) containing $1 \%$ protease inhibitor Phenylmethylsulfonyl Fluoride (PMSF) (Roche, Basel, Switzerland), followed by protein denaturation at $100{ }^{\circ} \mathrm{C}$ for 5 minutes. Afterwards, equal amounts of protein were separated by $10 \%$ sodium dodecyl sulfate-polyacrylamide gel electrophoresis (SDSPAGE) and electrorotated to polyvinylidene difluoride (PVDF) membranes. After blocking of the membranes with $5 \%$ skim milk for 120 minutes, the corresponding primary anti-DUSP14 (ab134265, Abcam, 1:1,000, Abcam, MA, USA), anti-Nrf2 (ab137550, Abcam, 1:1,000), anti-NF-

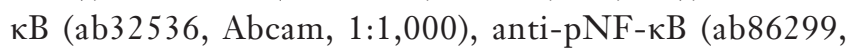

Abcam, 1:1,000), anti-Bax (ab32503, Abcam, 1:1,000), antiBcl2 (ab32124, Abcam, 1:1,000), anti-caspase-3 (ab13847, Abcam, 1:1,000), and anti-GAPDH (ab8245, Abcam, $1: 1,000)$ antibodies were added, and the membranes were incubated overnight at $4{ }^{\circ} \mathrm{C}$, after which the membranes were washed, supplemented with the matched secondary antibody, and incubated at room temperature for 120 minutes. Finally, the developing solution was added dropwise for development and band observation. GAPDH served as an internal reference. The experiment was repeated 3 times.

\section{Detection of oxidative stress markers}

Enzyme-linked immunosorbent assay (ELISA) was used to determine malondialdehyde (MDA) level, superoxide dismutase (SOD) activity, and glutathione peroxidase (GSHPX) content. The assay was performed strictly according to the instructions supplied with the reagent. Total thiol (TT) was determined using Dithio Dinitrobenzoic acid (DTNB) as a reducing agent. Afterward, $1 \mathrm{~mL}$ of trisEDTA (pH 8.6) buffer solution was added to $50 \mu \mathrm{L}$ of kidney tissue homogenate or cell homogenate, and the volume was determined at $412 \mathrm{~nm}$ by ultraviolet (UV) spectrophotometry. Subsequently, $20 \mu \mathrm{L}$ of DTNB reagent $(10 \mathrm{mmol} / \mathrm{L}$ solution) was poured into the above solution for 15 minutes before redetermination, and the concentration was calculated according to the ratio absorbance. Detection kits for the above oxidative stress markers were purchased from NanJing JianCheng Bioengineering Institute.

\section{Detection of cytokine levels}

First, 50-100 mg of kidney tissue was placed into a centrifuge tube, and then $0.01 \mathrm{~mol}$ PBS buffer was added at $0.05 \mathrm{~g} / \mathrm{mL}$. Following that, an ultrasonic cell pulverizer was used to make a milky white cell suspension. After 
centrifugation of the cell suspension at $12,000 \mathrm{r} / \mathrm{min}$ for 10 minutes, the supernatants were collected, or the supernatants of the cultured cells in each group were harvested for indicator determination. Finally, an ELISA kit (Abcam, Shanghai, China) was employed to verify the tissue suspension or cell culture supernatant factors IL-1 $1 \beta$, IL-6, and TNF- $\alpha$ in strict accordance with the manufacturer's instructions.

\section{Immunochemistry}

After treatment, kidney tissues resected from the rats were fixed in $10 \%$ paraformaldehyde and embedded in paraffin. Then, the tissue sections were got, dewaxed, hydrated, and washed 3 times with PBS for 3 minutes each time. The sections were placed in boiled citric acid repair solution under high pressure for 90 seconds and cooled naturally. Following that, the tissues were washed with PBS 3 times for 3 minutes each time, and immersed in $30 \mathrm{~mL} / \mathrm{L}$ of $\mathrm{H}_{2} \mathrm{O}_{2}$ at room temperature for 30 minutes for endogenous peroxidase inactivation. The tissues were again washed 3 times with PBS for 3 minutes each time, and then blocked with blocking buffer at room temperature for 30 minutes. Later on, diluted primary antibodies of anti-DUSP14 antibody (ab134265, Abcam, 1:200, Abcam, MA, USA) or anti-Caspase3 (ab13847, Abcam, 1:200) were added and incubated with the sections overnight at $4{ }^{\circ} \mathrm{C}$. After that, the sections were washed 3 times with PBS for 5 minutes each time, supplemented with horseradish peroxidase (HRP)labeled goat anti-mouse secondary antibody $(1: 5,000)$, and incubated for 1 hour at room temperature. Once more, the tissues were rinsed with PBS 3 times for 5 minutes each time, and diaminobenzidine (DAB) was added for color development. Finally, the tissues were rinsed with tap water, stained with hematoxylin, dehydrated, cleared, and mount observed under a microscope.

\section{Hematoxylin-eosin staining}

After treatment, rat kidney tissues were resected, fixed in paraformaldehyde, and embedded in paraffin. Then, the tissues were sliced into $4-\mu$ m-thick sections, which were baked in a constant temperature oven for 6 hours at $65^{\circ} \mathrm{C}$. Finally, the tissue sections were routinely dewaxed, hydrated, stained with hematoxylin and eosin (HE) staining, mounted with neutral balsam, and observed under a microscope.

\section{Cellular immunofluorescence}

After the completion of treatment, the HK-2 cell culture medium was discarded, and the cells were washed 3 times with PBS. After fixing with $10 \%$ paraformaldehyde, the cells were permeabilized and blocked in $5 \%$ goat serum. Next, the cells were incubated with primary anti-DUSP14 antibody (ab134265, Abcam, 1:100, Abcam, MA, USA) overnight at $4{ }^{\circ} \mathrm{C}$. After being washed with PBS, the cells were incubated at room temperature with secondary antibody conjugated with Alexa Fluor 594 for 1 hour, washed with PBS, and stained with 4',6-diamidino-2phenylin-dole (DAPI) (Beyotime Biotechnology, Shanghai, China). Finally, immunofluorescence images of the cells were captured using an inverted fluorescence microscope (Olympus, Tokyo, Japan).

\section{Data analysis}

Statistical analysis was performed using SPSS 21.0 (IBM Corp, Armonk, NY, USA). Measurement data were expressed as the mean \pm standard deviation. The $t$-test was used to compare the differences between 2 groups, while one-way analysis of variance (ANOVA) was used to compare the differences between multiple groups. $\mathrm{P}<0.05$ was considered to be statistically significant.

\section{Results}

\section{Eriocitrin abated OGD/R-induced apoptosis of HK-2 cells}

To explore the role of eriocitrin in IR-induced AKI, we constructed an OGD/R model of HK-2 cells administered different concentrations of eriocitrin. The results showed that HK-2 cell viability was weakened after OGD/R treatment $(\mathrm{P}<0.05$, Figure $1 A)$. Besides, OGD/R treatment also induced a higher apoptosis rate (Figure 1B,C), increased expression of caspase- 3 and Bax, and Bcl2 downregulation (Figure 1D). However, treatment with eriocitrin inhibited the OGD/R-induced decrease in cell viability and inhibited the OGD/R-induced apoptosis in a dose-dependent manner (Figure 1A,B,C,D). These results suggested that eriocitrin protected HK-2 cells from the OGD/R-induced injury.

\section{Eriocitrin distinctly attenuated OGD/R-induced inflammatory response and oxidative stress in $\mathrm{HK}-2$ cells}

To further explore the effects of eriocitrin on OGD/ 
A

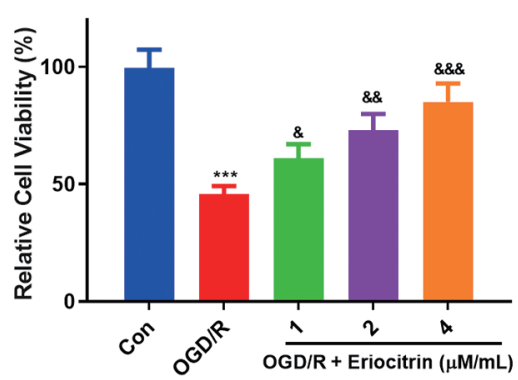

C

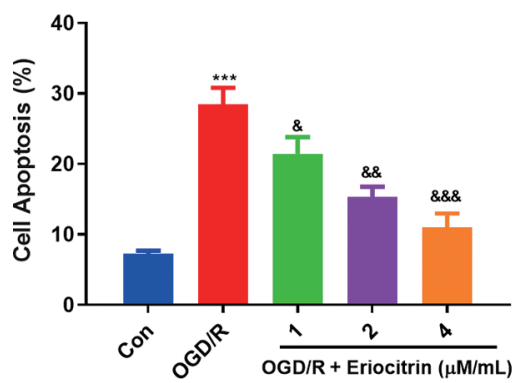

B
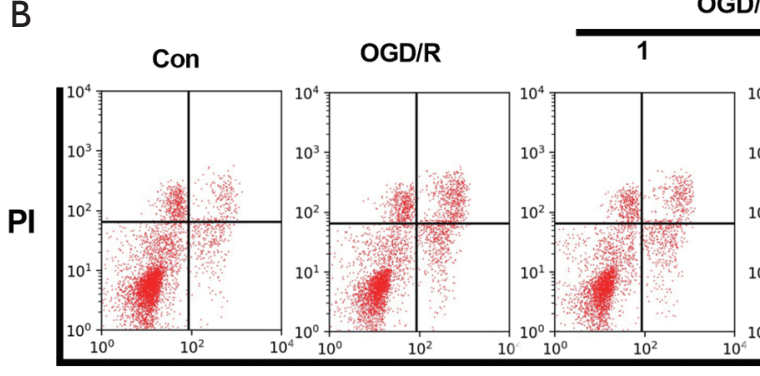

$O G D / R+$ Eriocitrin $(\mu \mathrm{M} / \mathrm{mL})$

D
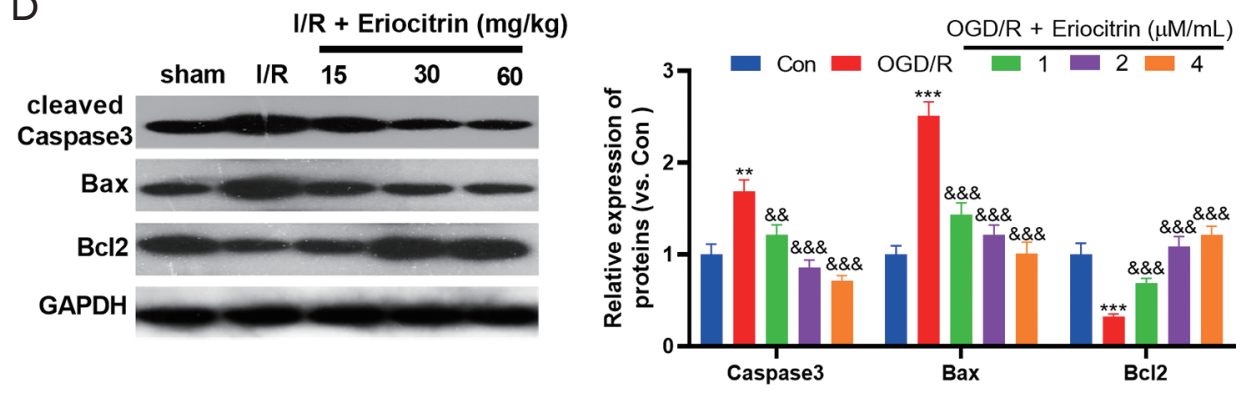

Figure 1 Eriocitrin reduced OGD/R-induced apoptosis in HK-2 cells. HK-2 cells were subjected to OGD/R and treated with different doses of eriocitrin (1-4 $\mu \mathrm{M})$. (A) CCK-8 assay was employed to detect HK-2 cells viability; (B,C) flow cytometry was performed to determine HK-2 cell apoptosis. (D) Apoptosis-related proteins including caspase-3, Bax, and Bcl2 were examined by western blot. ${ }^{* *} \mathrm{P}<0.01$, ${ }^{* * *} \mathrm{P}<0.001$ vs. Con group; ${ }^{\circledR} \mathrm{P}<0.05,{ }^{* \&} \mathrm{P}<0.01,{ }^{* \& \&} \mathrm{P}<0.001$ vs. OGD/R group. $\mathrm{N}=3$. OGD/R, oxygen glucose deprivation/reperfusion; IR, ischemia reperfusion.
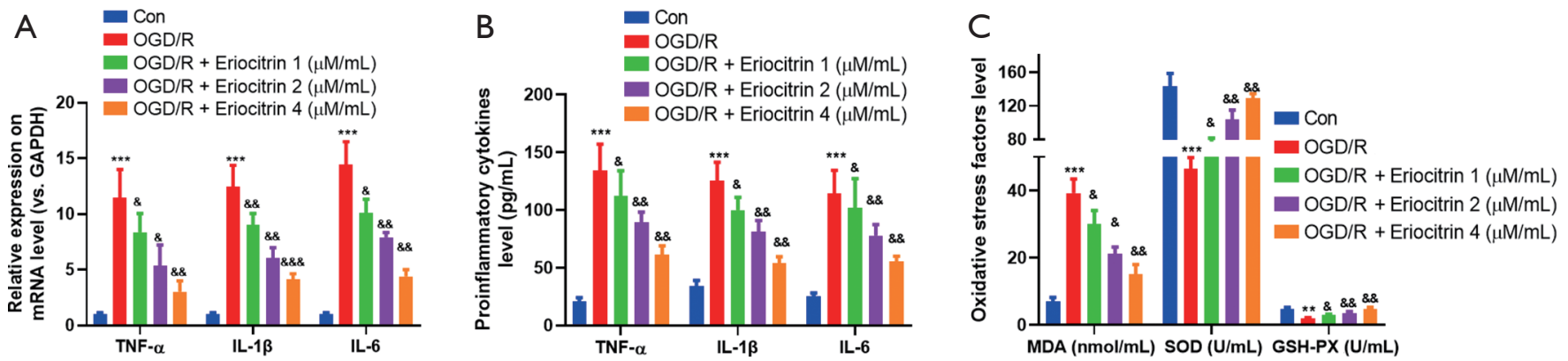

Figure 2 Eriocitrin notably attenuated OGD/R-induced inflammatory response and oxidative stress in HK-2 cells. HK-2 cells were subjected to OGD/R and treated with different doses of eriocitrin $(1-4 \mu M)$. (A,B) RT-PCR (A) and ELISA (B) were performed to determine the expression levels of IL-1 $\beta$, TNF- $\alpha$, and IL-6. (C) ELISA was employed to detect the expression of MDA, SOD, and GSH-PX in each group. ${ }^{* * *} \mathrm{P}<0.001$ vs. Con group; ${ }^{\&} \mathrm{P}<0.05,{ }^{{ }^{*} \times} \mathrm{P}<0.01,{ }^{\text {\&\&\& }} \mathrm{P}<0.001$ vs. OGD/R group. $\mathrm{N}=3$. OGD/R, oxygen glucose deprivation/ reperfusion; TNF- $\alpha$, tumor necrosis factor- $\alpha$; IL-1 $\beta$, interleukin $1 \beta$; IL-6, interleukin-6; MDA, malondialdehyde; SOD, superoxide dismutase; RT-PCR, real-time polymerase chain reaction.

$\mathrm{R}$-induced inflammatory response and oxidative stress in HK-2 cells, we adopted RT-PCR and ELISA to verify the expression of the pro-inflammatory cytokines IL$1 \beta$, TNF- $\alpha$, and IL-6. We found that the expression of the above pro-inflammatory factors in HK-2 cells was markedly increased by $\mathrm{OGD} / \mathrm{R}$, but were considerably inhibited by eriocitrin in a dose-dependent manner $(\mathrm{P}<0.05$, Figure $2 A, B)$. Further, the oxidative stress response in each 
A

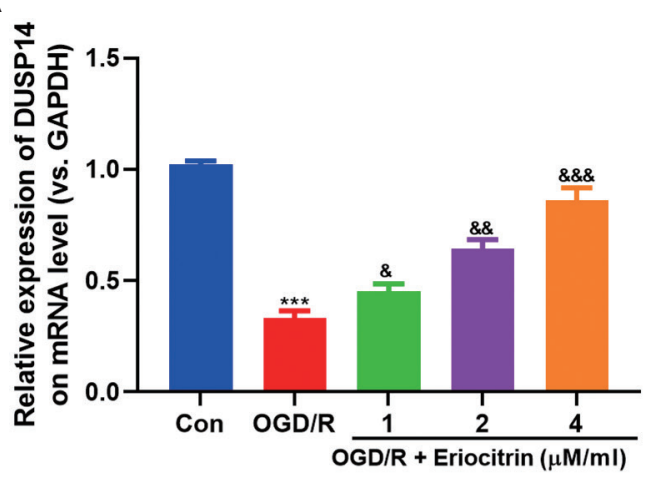

B

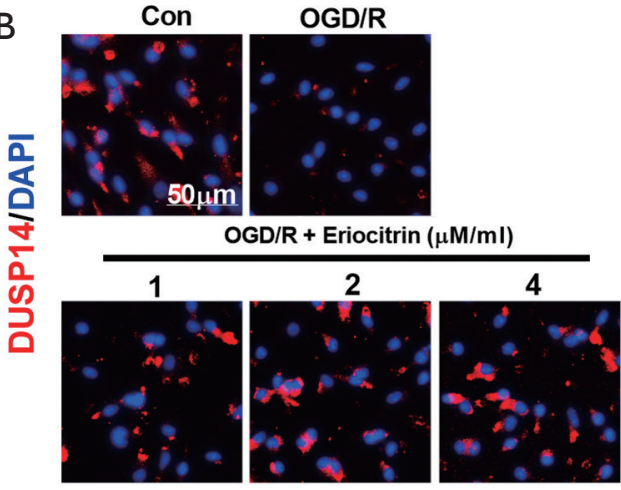

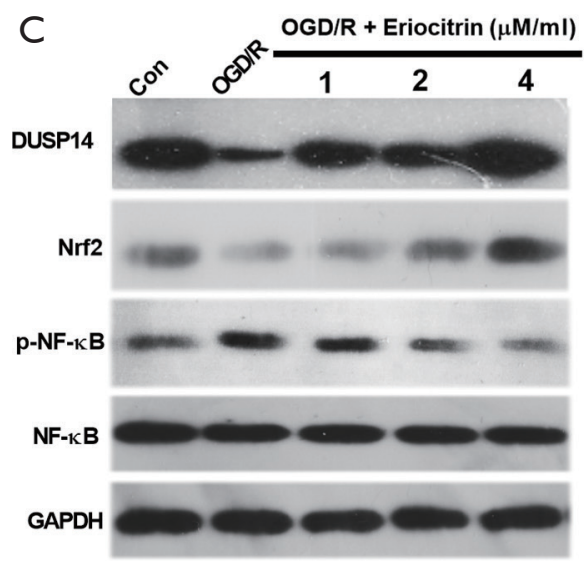

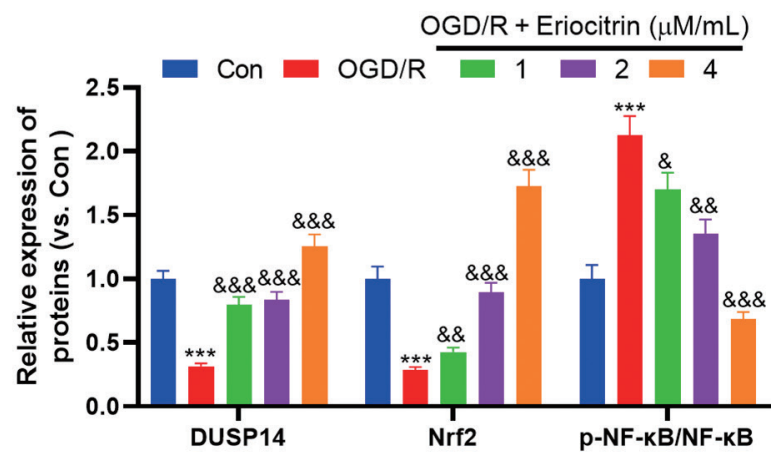

Figure 3 Eriocitrin modulated the Nrf2 and NF-kB pathway and upregulated DUSP14. HK-2 cells were subjected to OGD/R and treated with different doses of eriocitrin (1-4 $\mu \mathrm{M})$. (A) RT-PCR was carried out to test DUSP14 expression at the mRNA level; (B) Cellular immunofluorescence was conducted to detect DUSP14 (marked by red color) in HK-2 cells. (C) Western blot was performed to examine the

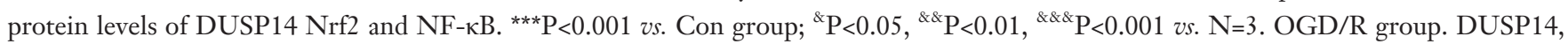
dual-specificity phosphatase 14; OGD/R, oxygen glucose deprivation/reperfusion; RT-PCR, real-time polymerase chain reaction; ELISA,

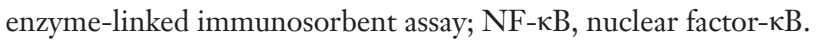

group was evaluated by ELISA. The results revealed that eriocitrin obviously inhibited OGD/R-induced MDA increasing and greatly enhanced the production of SOD and GSH-PX $(\mathrm{P}<0.05$, Figure $2 C)$. These results illustrated that eriocitrin notably alleviated OGD/R-induced inflammatory response and oxidative stress in HK-2 cells.

\section{Eriocitrin upregulated DUSP14 and Nrf2, and

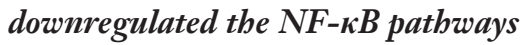

Next, we further explored the mechanism underlying the effect of eriocitrin on HK-2 cells. First, we found that OGD/R treatment markedly repressed DUSP14 expression, as detected by RT-PCR (Figure $3 A$ ), immunofluorescence (Figure $3 B$ ) and western blot (Figure 3C). Furthermore,
OGD/R treatment reduced the level of $\mathrm{Nrf} 2$ and activated the NF- $\kappa \mathrm{B}$ pathway in a concentration-dependent manner (Figure 3C). Eriocitrin also promoted DUSP14 expression, both at the mRNA and the protein level (Figure $3 A, B, C$ ). These results demonstrated that eriocitrin may promote $\mathrm{Nrf2}$ expression and inactivate the NF- $\mathrm{BB}$ pathway via upregulation of DUSP14.

\section{Inbibiting DUSP14 reversed the protective effects of eriocitrin on $\mathrm{HK}-2$ cells}

To explore whether the inhibition of DUSP14 reversed the protective effects of eriocitrin in HK-2 cells, we used PTP inhibitor IV, a DUSP14-specific inhibitor, to downregulate DUSP14. The ELISA results showed that PTP inhibitor 

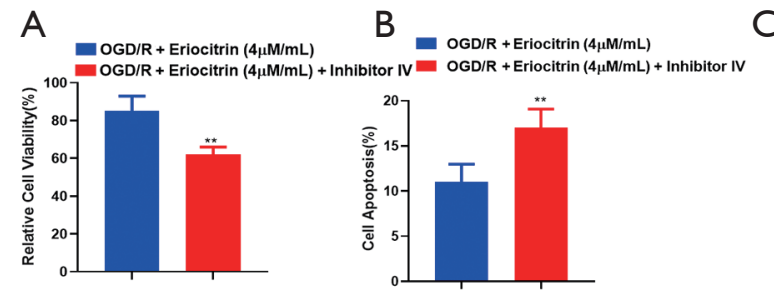
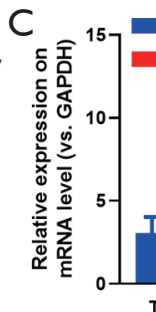

OGD/R + Eriocitrin $(4 \mu \mathrm{M} / \mathrm{mL})$ OGD/R + Eriocitrin $(4 \mu \mathrm{M} / \mathrm{mL})+$ Inhibitor IV ( Eriocitrin $(4, M / m L)+$ Inhibitor IV

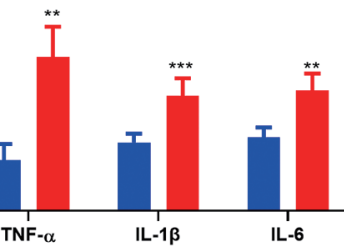

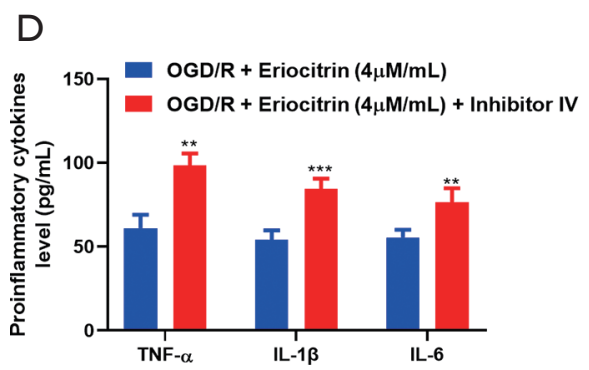

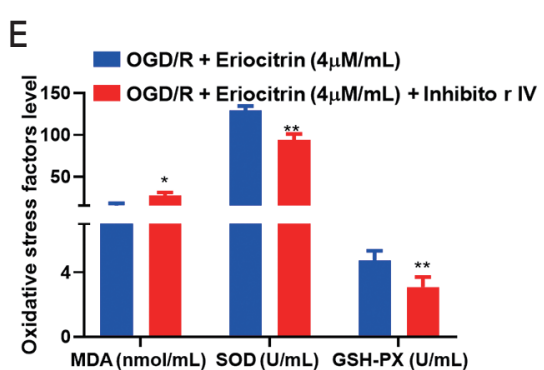

$\mathrm{F}$

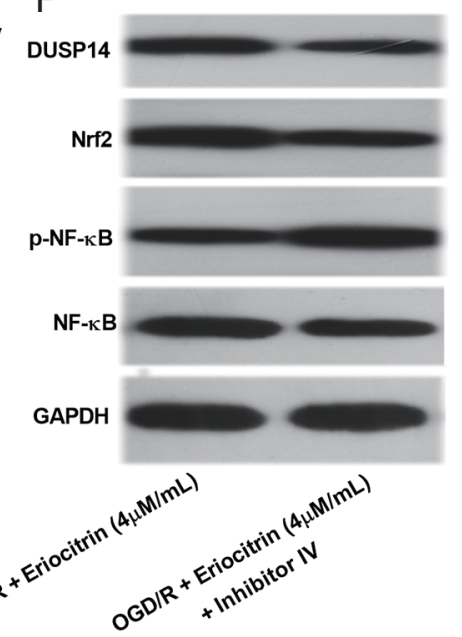

Figure 4 Inhibiting DUSP14 reversed the protective effect of eriocitrin on HK-2 cells. HK-2 cells were subjected to OGD/R and treated with different doses of eriocitrin $(4 \mu \mathrm{M})$ and/or PTP inhibitor IV (50 Mm). (A,B). CCK-8 and flow cytometry were used to test the viability (A) and apoptosis (B) of HK-2 cells. (C,D) RT-PCR (C) and ELISA (D) were performed to test the effect of PTP inhibitor IV on the expression of IL-1 $\beta$, TNF- $\alpha$, and IL-6. D ELISA was carried out to examine changes in the levels of MDA, SOD, and GSH-PX. (E,F) Changes in

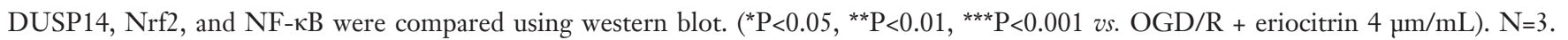
OGD/R, oxygen glucose deprivation/reperfusion; DUSP14, dual-specificity phosphatase 14; IR, ischemia reperfusion; PTP, protein tyrosine phosphatase; RT-PCR, real-time polymerase chain reaction; ELISA, enzyme-linked immunosorbent assay; IL- $1 \beta$, interleukin $1 \beta$; TNF- $\alpha$, tumor necrosis factor- $\alpha$; IL-6, interleukin-6; MDA, malondialdehyde; SOD, superoxide dismutase.

IV reversed the effects of eriocitrin by weakening the viability and increasing apoptosis of HK-2 cells (Figure 4A). Meanwhile, the results of RT-PCR and ELISA also proved that PTP inhibitor IV markedly increased the expression of the pro-inflammatory factors IL- $1 \beta$, TNF- $\alpha$, and IL- 6 compared with the OGD/R + eriocitrin group $(\mathrm{P}<0.05$, Figure $4 B, C)$. Besides, PTP inhibitor IV also increased the level of MDA, while greatly attenuating the levels of SOD and GSH-PX $(\mathrm{P}<0.05$, Figure $4 D)$. Moreover, western blot was conducted to explore the changes of Nrf2 and NF- $\kappa \mathrm{B}$. PTP inhibitor IV was observed to distinctly abate Nrf2 expression and activate NF- $\kappa \mathrm{B}$ signalling pathway $(\mathrm{P}<0.05$, Figure $4 E, F)$. These results demonstrated that eriocitrin alleviated the injury of HK-2 cells in the OGD/R model dose-dependently through upregulation of DUSP14.

\section{Eriocitrin attenuated I/R-induced apoptosis and kidney damage}

To further verify the protective effects of eriocitrin against AKI, we established an I/R-induced AKI model in rats and administered the rats with different concentrations of eriocitrin. Surprisingly, the results showed that eriocitrin protected kidney function and diminished the levels of BUN and serum creatinine in rat serum $(\mathrm{P}<0.05$, Figure $5 A)$. Besides, eriocitrin also reduced glomeruli and tubule damage $(\mathrm{P}<0.05$, Figure $5 B)$ and apoptosis $(\mathrm{P}<0.05$, Figure $5 C, D)$ in a dose-dependent manner. These observations indicated that eriocitrin exerted protective effects against IR-induced AKI.

\section{Eriocitrin notably inbibited IR-mediated inflammation and oxidative stress in kidney tissues}

To further explore the effects of eriocitrin on IR-induced kidney inflammation and oxidative stress, RT-PCR and ELISA were performed to compare the alterations in the expression of the pro-inflammatory factors IL- $1 \beta$, TNF- $\alpha$, and IL-6. The results demonstrated that the expression levels of the above pro-inflammatory factors were distinctly enhanced in kidney tissues following IR, but were dosedependently reduced by eriocitrin treatment $(\mathrm{P}<0.05$, Figure $6 A, B)$. In addition, oxidative stress response was evaluated in each group by ELISA, and the results showed 
A
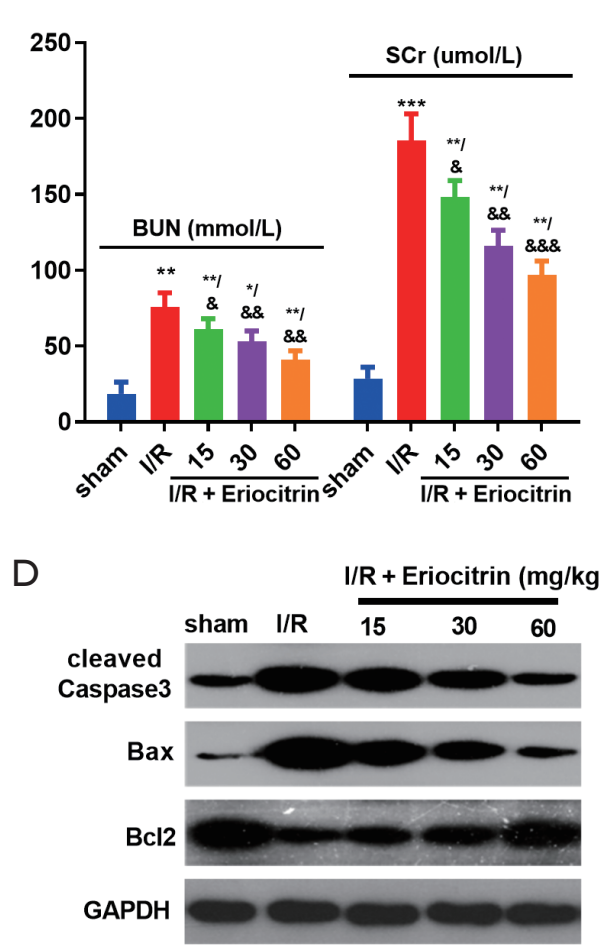

B
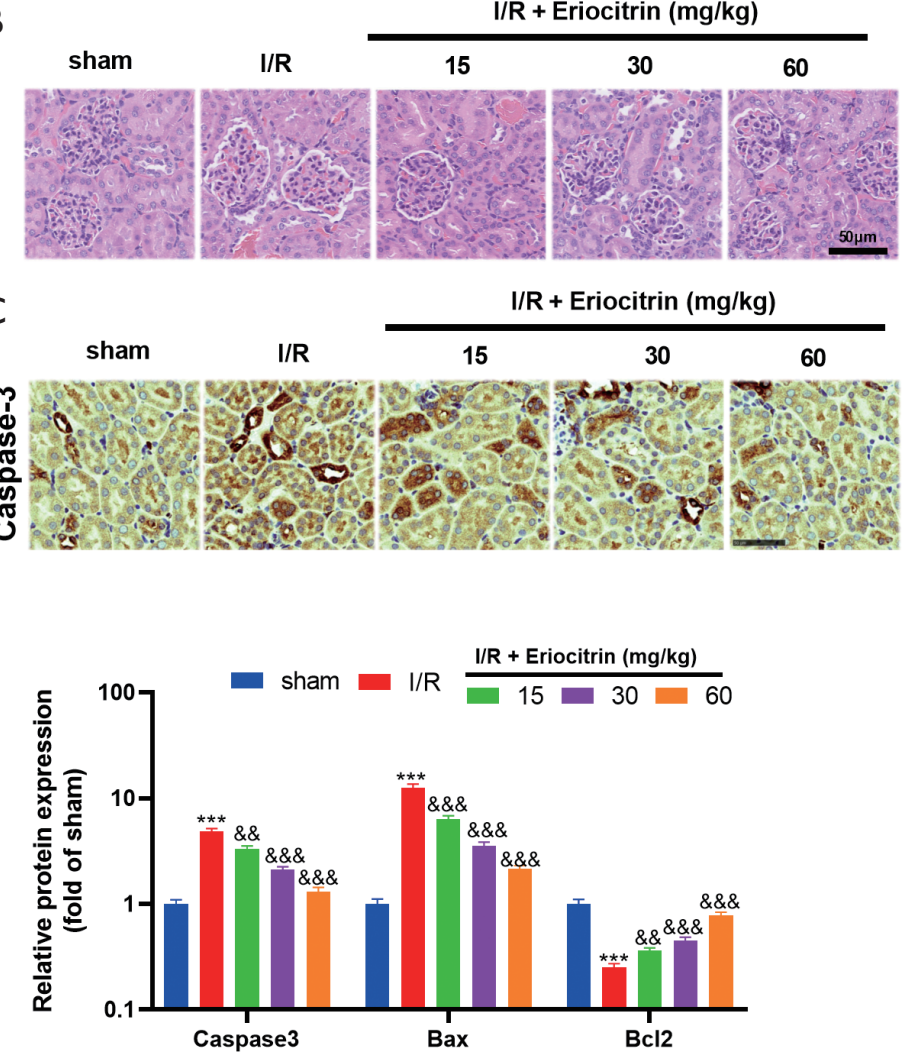

Figure 5 Eriocitrin reduced IR-induced apoptosis and kidney injury. An IR-induced kidney injury model was established using male SD rats. The rats were treated with eriocitrin at a high dose $(60 \mathrm{mg} / \mathrm{kg})$, medium dose $(30 \mathrm{mg} / \mathrm{kg})$, or low dose $(15 \mathrm{mg} / \mathrm{kg})$. (A) ELISA was used to detect the levels of BUN and creatinine in the serum. (B) HE staining was employed to detect glomerular and tubule damage in the different groups. (C) Immunohistochemical staining was performed to determine cell apoptosis (marked by caspase-3) in each group, the scale bar was $50 \mu \mathrm{M}$. (D) Apoptosis-related proteins including caspase-3, Bax, and Bcl 2 were detected by western blot. ${ }^{*} \mathrm{P}<0.05,{ }^{* *} \mathrm{P}<0.01,{ }^{* * *} \mathrm{P}<0.001$ vs. sham group; ${ }^{{ }^{8} \mathrm{P}<0.05}$, ${ }^{\text {\&\& }} \mathrm{P}<0.01,{ }^{\text {\&\&\& }} \mathrm{P}<0.001$ vs. $\mathrm{N}=10$. IR group. IR, ischemia reperfusion; SD, Sprague-Dawley; ELISA, enzyme-linked immunosorbent assay; RT-PCR, real-time polymerase chain reaction.

that eriocitrin markedly inhibited IR-induced MDA expression, while notably elevating the expression of SOD and GSH-PX (vs. I/R group) (Figure 6C). These results demonstrated that eriocitrin alleviates the inflammatory response and oxidative stress in kidney tissues.

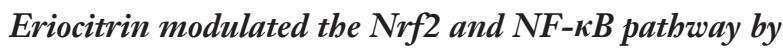 upregulating DUSP14}

Next, we carried out RT-PCR, western blot, and immunohistochemistry to detect the expression changes of DUSP14 in kidney tissues under eriocitrin treatment. After IR, DUSP14 expression was remarkably inhibited in kidney tissues but was increased by eriocitrin (Figure $7 A, B, C)$. In addition, eriocitrin significantly enhanced $\mathrm{Nrf} 2$ expression and inactivated $\mathrm{NF}-\kappa \mathrm{B}$ signalling pathway (Figure $7 \mathrm{C}$ ). These results showed that eriocitrin promotes $\mathrm{Nrf2}$ expression and significantly attenuates $\mathrm{NF}-\mathrm{\kappa B}$ activation by upregulating DUSP14.

\section{DUSP14 inhibition reversed the protective effects of eriocitrin on kidney function}

To investigate whether inhibiting DUSP14 could reverse the protective effects of eriocitrin in IR-induced AKI, we used PTP inhibitor IV to diminish DUSP14 expression. Interestingly, after the administration of PTP inhibitor IV, kidney functions were more worse (Figure 8A), accompanied with enhanced pro-inflammatory and oxidative stress responses (Figure 8B,C,D). Moreover, the DUSP14 and 

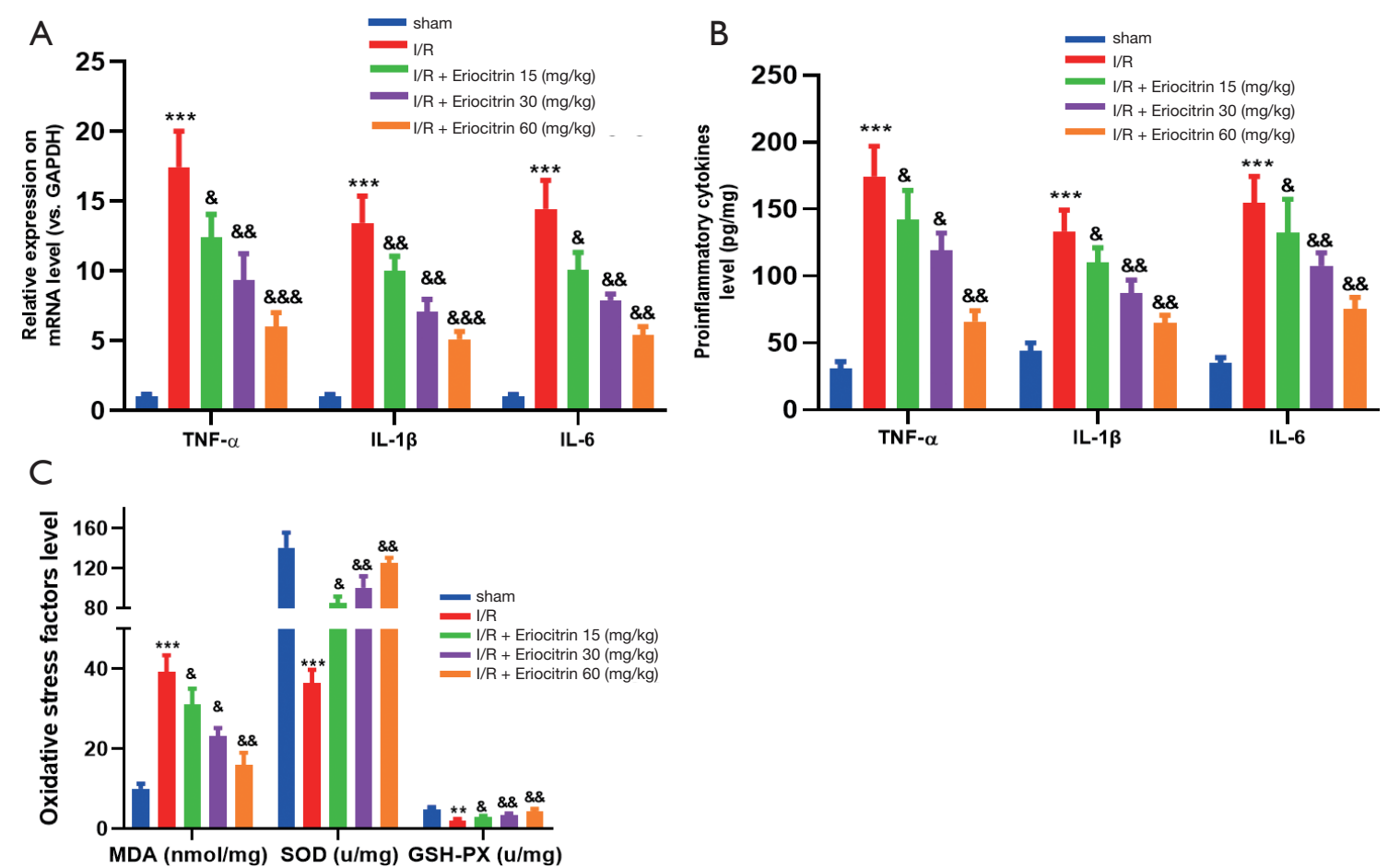

Figure 6 Eriocitrin attenuated inflammation and oxidative stress IR-mediated renal tissue. An IR-induced kidney injury model was established using male SD rats. The rats were treated with eriocitrin at a high dose $(60 \mathrm{mg} / \mathrm{kg})$, medium dose $(30 \mathrm{mg} / \mathrm{kg})$, or low dose $(15 \mathrm{mg} / \mathrm{kg})$. (A,B) RT-PCR (A) and ELISA (B) were employed to detect the expressive changes of IL-1 $\beta$, TNF- $\alpha$, and IL-6 in rat kidney tissues. (C) ELISA was carried out to compare the expression levels of MDA, SOD, and GSH-PX in each group. ${ }^{* * *} \mathrm{P}<0.001$ vs. sham group; ${ }^{\&} \mathrm{P}<0.05,{ }^{\& \&} \mathrm{P}<0.01,{ }^{\& \& \&} \mathrm{P}<0.001$ vs. IR group. $\mathrm{N}=10$. IR, ischemia reperfusion; RT-PCR, real-time polymerase chain reaction; TNF- $\alpha$, tumor necrosis factor- $\alpha$; IL-1 $\beta$, interleukin $1 \beta$; IL-6, interleukin-6; MDA, malondialdehyde; SOD, superoxide dismutase.

Nrf2 levels in kidney tissues were downregulated and $\mathrm{NF}-\kappa \mathrm{B}$ phosphorylation was elevated compared with the $\mathrm{IR}+$ eriocitrin group (Figure $8 E, F$ ). The above results demonstrated that eriocitrin alleviates IR-induced AKI, mainly by upregulating DUSP14.

\section{Discussion}

IR is a common cause of AKI, which is characterized by ARF. Mounting evidence suggests that inflammation and oxidative stress are the primary causes of IR-induced AKI, and anti-inflammation and anti-oxidative therapies are its main treatment methods (13). Eriocitrin has been found to exert an anti-inflammatory function in multiple human inflammatory diseases (14). Here, we first demonstrated that eriocitrin increased Nrf-2 expression and inactivates NF- $\mathrm{NB}$ by upregulating DUSP14, thereby attenuating apoptosis, inflammation, and oxidative stress in IR-mediated renal injury in rats.
At present, there are many reports about the effects of Chinese herbal extracts on IR-induced AKI. For instance, Geyikoglu et al. reported that propolis and its combination with boric acid distinctly abated IR-mediated kidney injury by inhibiting oxidative stress and inflammation in kidney tissues to attenuate cellular DNA injury and apoptosis (15). Furthermore, Topdağ $\mathrm{et}_{\text {et }}$ l. revealed that preventive markedly alleviated IR-mediated oxidation and inflammation in kidney tissues, and increased the level of antioxidant 8-OHdG (16). In contrast, Najafi et al. reported that Malva sylvestris L. notably reduced leukocyte invasion, decreased the expression of TNF-1 and ICAM-1 mRNA in tissues, and alleviated oxidative stress, thereby protecting the kidney from IR injury (17). Some other scholars have reported that extracts from garlic (18) and saffron (19) can also prevent IR induced AKT. Thus, it can be seen that natural extracts are effective in alleviating IR-mediated oxidative stress and inflammation in kidney tissues.

Eriocitrin is also called eriodictyol 7-O-beta-rutinoside. 

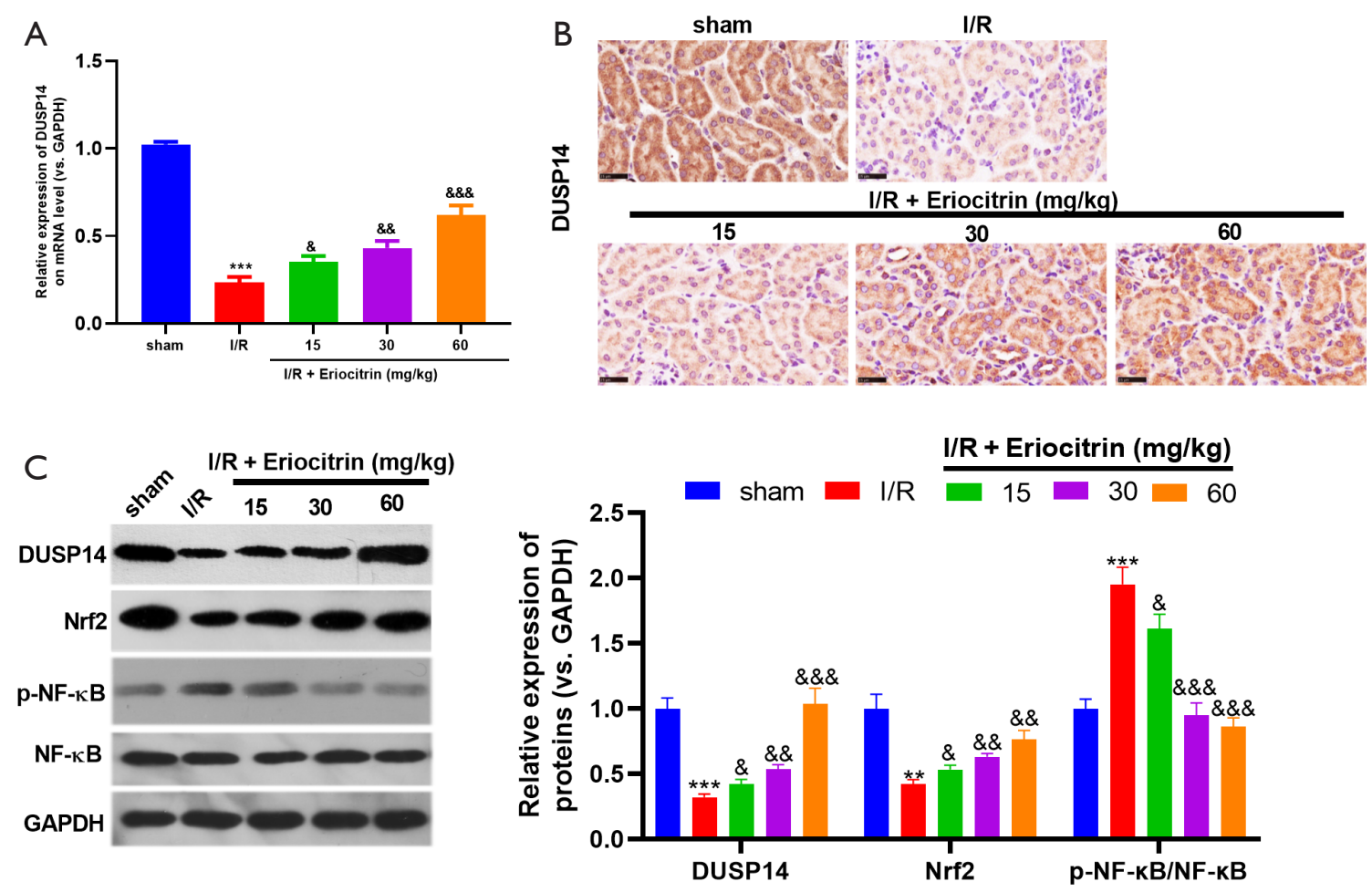

Figure 7 Eriocitrin modulated the Nrf2 and NF- $\mathrm{B}$ pathway by upregulating DUSP14. An IR-induced kidney injury model was established using male SD rats. The rats were treated with eriocitrin at a high dose (60 mg/kg), medium dose (30 mg/kg), or low dose (15 mg/kg). (A) RT-PCR was used to test the DUSP14 mRNA levels in the kidney tissues. (B) IHC was performed to detect DUSP14 expression, the scale bar was $25 \mu \mathrm{M}$. (C) Western blot was carried out to examine the expressions of DUSP14, Nrf2, and NF- $\mathrm{B}$ in the kidney tissues. ** $\mathrm{P}<0.01$, ${ }^{* * *} \mathrm{P}<0.001$ vs. sham group; ${ }^{*} \mathrm{P}<0.05,{ }^{\& \&} \mathrm{P}<0.01$, ${ }^{\& \& \&} \mathrm{P}<0.001$ vs. IR group. $\mathrm{N}=10$. DUSP14, dual-specificity phosphatase $14 ;$ IR, ischemia

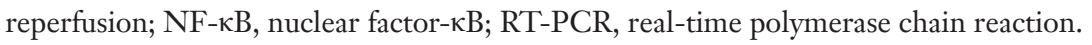

Its chemical formula is $\mathrm{C} 27 \mathrm{H} 33 \mathrm{O} 16$, and its molecular structure is shown in Sup-1. Eriocitrin is a stronger antioxidant than the other citrus flavonoid compounds, and it is abundant in lemon and lime, with its safety evidenced by the lack of developmental toxicity in zebrafish (20). In recent years, the anti-inflammatory qualities of eriocitrin have been increasingly reported. For instance, Hiramitsu et al. reported that eriocitrin weakened diet-induced hepatic steatosis by activating mitochondria transcription factor, nuclear respiratory factor, cytochrome coxidase subunit, and ATP synthase (21). Meanwhile, Shen et al. described eriocitrin as being the main component of Chinese crude polyphenols (CAVAP-W). In addition, CAVAP-W was found to markedly dampen the inflammatory response and oxidative stress, and inactivate the nitric oxide synthase (iNOS), IL-6, TNF- $\alpha$, IL-1 $\beta$, and cyclooxygenase-2 (COX-2) in Lipopolysaccharides (LPS)-treated RAW264.7 cells (22). On the other hand, Ferreira et al. confirmed that eriocitrin increases the total antioxidant capacity of serum, and restrains the facilitation of IL-6, macrophage chemoattractant protein-1 (MCP-1), and C-reactive protein (hs-CRP), thus preventing systemic inflammation and alleviating oxidative stress in C57BL/6J mice fed with highfat diets (23). Besides, there have been many reports on the anti-oxidative effects of eriocitrin. For instance, Miyake reported that eriocitrin reduced the levels of oxidative stress molecule 8-hydroxydeoxyguanosine and exerts a protective effect in diabetic rats (24). Likewise, Minato et al. found that eriocitrin can obviously decrease the levels of liver oxidative stress molecules $\mathrm{N}$ (epsilon)-(hexanoyl) lysine, o, o-dityrosine, and nitrotyrosine, thereby exerting a protective function (5). The results of the current study are consistent with those reported previously. Specifically, we observed that eriocitrin downregulated the levels of the inflammatory response factors IL- $1 \beta$, TNF- $\alpha$, and IL- 6 in both in vivo and in vitro experiments of AKI, inhibited the 

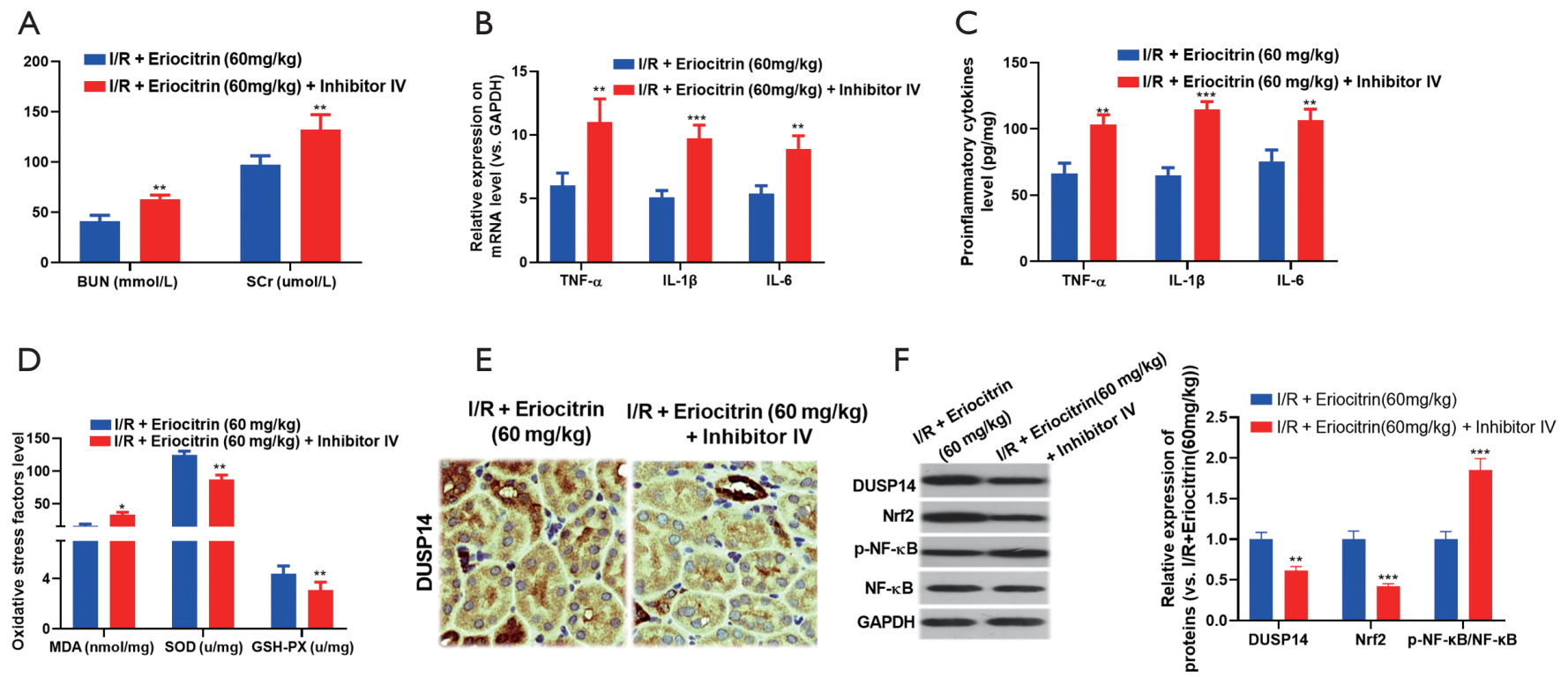

Figure 8 Inhibiting DUSP14 reversed the renal protective effects of eriocitrin. An IR-induced kidney injury model was established using male SD rats. The rats were treated with eriocitrin $(60 \mathrm{mg} / \mathrm{kg})$ and/or PTP inhibitor IV (5 mg/kg). (A) ELISA was employed to detect the levels of BUN and creatinine in rat serum. (B,C) RT-PCR (B) and ELISA (C) were employed to examine IL-1 $\beta$, TNF- $\alpha$ and IL- 6 expression. (D) ELISA was carried out to detect the levels of MDA, SOD and GSH-PX in serum. (E) IHC was performed to detect DUSP14 expression, the scale bar was $25 \mu \mathrm{M}$. (F) Western blot was used to explore changes in DUSP14, Nrf2, and NF- $\mathrm{B}$ expression. ${ }^{*} \mathrm{P}<0.05,{ }^{* *} \mathrm{P}<0.01,{ }^{* *} \mathrm{P}<0.001$ vs. I/R + eriocitrin $60 \mathrm{mg} / \mathrm{kg}$ group. $\mathrm{N}=10$. DUSP14, dual-specificity phosphatase $14 ;$ IR, ischemia reperfusion; SD, Sprague-Dawley; PTP, protein tyrosine phosphatase; ELISA, enzyme-linked immunosorbent assay; RT-PCR, real-time polymerase chain reaction; IL- $1 \beta$, interleukin $1 \beta$; TNF- $\alpha$, tumor necrosis factor- $\alpha$; IL-6, interleukin-6; MDA, malondialdehyde; SOD, superoxide dismutase; NF- $\kappa$ B, nuclear factor- $\kappa \mathrm{B}$.

expression of the oxidation factor MDA, and increased the levels of the anti-peroxidation factor SOD and GSH-PX, thereby preventing IR-induced AKI.

It is now recognized that IR directly activates JNK and $\mathrm{P} 38$, and eventually activates $\mathrm{NF}-\kappa \mathrm{B}$, resulting in inflammatory response and oxidative stress. Additionally, numerous studies have demonstrated that inhibiting $\mathrm{NF}-\kappa \mathrm{B}$ reduces kidney damage in IR-induced AKI (25). Some reports have also revealed that Nrf-2 expression is obviously diminished in kidney injury, and upregulating Nrf-2 alleviates kidney injury by repressing oxidative stress $(26,27)$. Therefore, the regulation of Nrf-2 and NF- $\mathrm{KB}$ may be an important factor affecting AKI. As an atypical DUSP, DUSP14 has been studied for its antioxidant and anti-inflammatory effects in IR. Previous report showed that DUSP14 dephosphorylates many inflammatory and oxidative stress singling pathways like MAPK, ERK)1/2 and p38 (7-10). For example, Song et al. reported that DUSP14 overexpression reduces the production of ROS and downregulates TNF- $\alpha$ and IL- $1 \beta$ by upregulating the Nrf-2 signaling pathway, thereby protecting mice from IR-induced cerebral damage (11). Meanwhile, Lin et al. verified that DUSP14 KD increases the activation of hypoxia and reoxygenation-induced NF- $\mathrm{KB}$ and MAPK signaling pathways as well as ROS production, suggesting that DUSP14 is a positive regulator of myocardial IR. Besides, inhibition of DUSP14 aggravates myocardial injury by activating ROS-regulated NF- $\kappa \mathrm{B}$ and MAPK signaling pathways (28). Moreover, Li et al. also confirmed that DUSP14 inactivates the NF- $\mathrm{BB}$ and MAPK pathways, thereby preventing IR injury (29). The above reports demonstrate that DUSP14 upregulates Nrf-2 and inhibits the NF- $\mathrm{\kappa B}$ pathway, thereby contributing to IR-mediated injury. Our report also confirmed for the first time that DUSP14 is obviously downregulated in IR-induced AKI, while its upregulation enhances Nrf-2 levels and inactivates the NF- $\mathrm{kB}$ pathway, thereby exerting a protective effect.

Disappointingly, the mechanism of eriocitrin's effect in IR-induced AKI remains elusive. Shen et al. reported

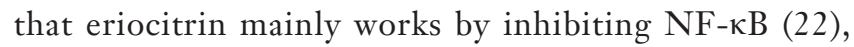


and our study further explored this mechanism. Through further exploration, we discovered that eriocitrin promotes Nrf-2 and inactivates NF- $\kappa$ B, mainly through DUSP14 upregulation. Moreover, using the DUSP14-specific inhibitor PTP inhibitor IV considerably reduced the protective effects of eriocitrin against AKI. Therefore, eriocitrin exerts anti-oxidant and anti-inflammatory effects by upregulating DUSP14.

In summary, our research confirmed that eriocitrin elevates DUSP14 expression to enhance Nrf-2 and inactivate the NF- $\mathrm{NB}$ pathway in IR-induced AKI, thereby attenuating oxidative stress and inflammation in rats to protect the kidney. Overall, our results suggest that eriocitrin has good application prospects for the treatment of IR-induced AKI.

\section{Acknowledgments}

Funding: None.

\section{Footnote}

Reporting Checklist: The authors have completed the ARRIVE reporting checklist. Available at http://dx.doi. org/10.21037/atm-21-337

Data Sharing Statement: Available at http://dx.doi. org/10.21037/atm-21-337

Conflicts of Interest: All authors have completed the ICMJE uniform disclosure form (available at http://dx.doi. org/10.21037/atm-21-337). The authors have no conflicts of interest to declare.

Ethical Statement: The authors are accountable for all aspects of the work in ensuring that questions related to the accuracy or integrity of any part of the work are appropriately investigated and resolved. All animal experiments were approved by the Ethics Committee of The Affiliated Baiyun Hospital of Guizhou Medical University. All animal operations were performed according to the National Institutes of Health Guide for the Care and Use of Laboratory Animals.

Open Access Statement: This is an Open Access article distributed in accordance with the Creative Commons Attribution-NonCommercial-NoDerivs 4.0 International License (CC BY-NC-ND 4.0), which permits the non- commercial replication and distribution of the article with the strict proviso that no changes or edits are made and the original work is properly cited (including links to both the formal publication through the relevant DOI and the license). See: https://creativecommons.org/licenses/by-nc-nd/4.0/.

\section{References}

1. Li XY, Zhang YQ, Xu G, et al. miR-124/MCP-1 signaling pathway modulates the protective effect of itraconazole on acute kidney injury in a mouse model of disseminated candidiasis. Int J Mol Med 2018;41:3468-76.

2. Machado-Aranda D, Suresh M, Yu B, et al. RNA From Necrotic Cells Drives the Acute Inflammatory Response and Injury Through Toll-Like Receptor-3 (TLR-3) Dependent Pathway in Lung Contusion. J Surg Res 2013;179:326.

3. Wang L, Wang Q, Sheng K, et al. A new graphene nanocomposite modified electrode as efficient voltammetric sensor for determination of eriocitrin. J Electroanal Chem 2017;785:96-102.

4. Guo G, Shi W, Shi F, et al. Anti-inflammatory effects of eriocitrin against the dextran sulfate sodium-induced experimental colitis in murine model. J Biochem Mol Toxicol 2019;33:e22400.

5. Minato K, Miyake Y, Fukumoto S, et al. Lemon flavonoid, eriocitrin, suppresses exercise-induced oxidative damage in rat liver. Life Sci 2003;72:1609-16.

6. Hijikata M, Matsushita I, Le Hang NT, et al. Influence of the polymorphism of the DUSP14 gene on the expression of immune-related genes and development of pulmonary tuberculosis. Genes Immun 2016;17:207-12.

7. Yang CY, Li JP, Chiu LL, et al. Dual-specificity phosphatase 14 (DUSP14/MKP6) negatively regulates TCR signaling by inhibiting TAB1 activation. J Immunol. 2014;192:1547-57.

8. Yang CY, Chiu LL, Tan TH. TRAF2-mediated Lys63linked ubiquitination of DUSP14/MKP6 is essential for its phosphatase activity. Cell Signal 2016;28:145-51.

9. Jin $\mathrm{Q}, \mathrm{Li} \mathrm{R}, \mathrm{Hu} \mathrm{N}$, et al. DUSP1 alleviates cardiac ischemia/reperfusion injury by suppressing the Mff-required mitochondrial fission and Bnip3-related mitophagy via the JNK pathways. Redox Biol 2018;14:576-87.

10. Oehrl W, Cotsiki M, Panayotou G. Differential regulation of M3/6 (DUSP8) signaling complexes in response to arsenite-induced oxidative stress. Cell Signal. 2013;25:429-38.

11. Jianrong S, Yanjun Z, Chen Y, et al. DUSP14 rescues 
cerebral ischemia/reperfusion (IR) injury by reducing inflammation and apoptosis via the activation of Nrf-2. Biochem Biophys Res Commun 2019;509:713-21.

12. Park JE, Park BC, Song M, et al. PTP inhibitor IV protects JNK kinase activity by inhibiting dual-specificity phosphatase 14 (DUSP14). Biochem Biophys Res Commun 2009;387:795-9.

13. Lorenzen JM. Vascular and circulating microRNAs in renal ischaemia-reperfusion injury. J Physiol 2015;593:1777-84.

14. Riachi LG, De Maria CA. Peppermint antioxidants revisited. Food Chem 2015;176:72-81.

15. Geyikoglu F, Koc K, Colak S, et al. Propolis and Its Combination with Boric Acid Protect Against Ischemia/ Reperfusion-Induced Acute Kidney Injury by Inhibiting Oxidative Stress, Inflammation, DNA Damage, and Apoptosis in Rats. Biol Trace Elem Res 2019;192:214-21.

16. Topdağ 1 Ö, Tanyeli A, Akdemir FNE, et al. Preventive effects of fraxin on ischemia/reperfusion-induced acute kidney injury in rats. Life Sci 2020;242:117217.

17. Najafi H, Mohamadi Yarijani Z, Changizi-Ashtiyani S, et al. Protective effect of Malva sylvestris L. extract in ischemia-reperfusion induced acute kidney and remote liver injury. PLoS One 2017;12:e0188270.

18. Ali SI, Alhusseini NF, Atteia HH, et al. Renoprotective effect of a combination of garlic and telmisartan against ischemia/reperfusion-induced kidney injury in obese rats. Free Radic Res 2016;50:966-86.

19. Mahmoudzadeh L, Najafi H, Ashtiyani SC, et al. Antiinflammatory and protective effects of saffron extract in ischaemia/reperfusion-induced acute kidney injury. Nephrology (Carlton) 2017;22:748-54.

20. Miyake Y, Yamamoto K, Morimitsu Y, et al. Isolation of C-glucosylflavone from lemon peel and antioxidative activity of flavonoid compounds in lemon fruit. J. Agric. Food Chem 1997;45:4619-23.

21. Hiramitsu M, Shimada Y, Kuroyanagi J, et al. Eriocitrin ameliorates diet-induced hepatic steatosis with activation of mitochondrial biogenesis. Sci Rep 2014;4:3708.

Cite this article as: $\mathrm{Xu} \mathrm{J}, \mathrm{Ma} \mathrm{L}, \mathrm{Fu}$ P. Eriocitrin attenuates ischemia reperfusion-induced oxidative stress and inflammation in rats with acute kidney injury by regulating the dual-specificity phosphatase 14 (DUSP14)-mediated Nrf2 and nuclear factor$\kappa \mathrm{B}(\mathrm{NF}-\kappa \mathrm{B})$ pathway. Ann Transl Med 2021;9(4):350. doi: 10.21037/atm-21-337
22. Shen CY, Jiang JG, Huang CL, et al. Polyphenols from Blossoms of Citrus aurantium L. var. amara Engl. Show Significant Anti-Complement and Anti-Inflammatory Effects. J Agric Food Chem 2017;65:9061-8.

23. Ferreira PS, Spolidorio LC, Manthey JA, et al. Citrus flavanones prevent systemic inflammation and ameliorate oxidative stress in C57BL/6J mice fed high-fat diet. Food Funct 2016;7:2675-81.

24. Miyake Y, Yamamoto K, Tsujihara N, et al. Protective effects of lemon flavonoids on oxidative stress in diabetic rats. Lipids 1998;33:689-95.

25. Zeng M, Qi M, Wang Y, et al. 5-O-methyldihydroquercetin and cilicicone B isolated from Spina Gleditsiae ameliorate lipopolysaccharideinduced acute kidney injury in mice by inhibiting inflammation and oxidative stress via the TLR4/MyD88/ TRIF/NLRP3 signaling pathway. Int Immunopharmacol 2020;80:106194.

26. Dizaji R, Sharafi A, Pourahmad J, et al. The effects of Hemiscorpius lepturus induced-acute kidney injury on PGC-1 $\alpha$ gene expression: From induction to suppression in mice. Toxicon 2020;174:57-63.

27. Amini N, Sarkaki A, Dianat M, et al. Protective effects of naringin and trimetazidine on remote effect of acute renal injury on oxidative stress and myocardial injury through Nrf-2 regulation. Pharmacol Rep 2019;71:1059-66.

28. Lin B, Xu J, Feng DG, et al. DUSP14 knockout accelerates cardiac ischemia reperfusion (IR) injury through activating $\mathrm{NF}-\kappa \mathrm{B}$ and MAPKs signaling pathways modulated by ROS generation. Biochem Biophys Res Commun 2018;501:24-32.

29. Li Y, Fei L, Wang J, et al. Inhibition of miR-217 Protects Against Myocardial Ischemia-Reperfusion Injury Through Inactivating NF- $\kappa \mathrm{B}$ and MAPK Pathways. Cardiovasc Eng Technol 2020;11:219-27.

(English Language Editor: J. Reynolds) 